\title{
openheart Influence of cardiovascular risk factors on infarct size and interaction with mechanical ischaemic postconditioning in ST-elevation myocardial infarction
}

Sophie Pichot, ${ }^{1}$ Nathan Mewton, ${ }^{1,2}$ Theodora Bejan-Angoulvant, ${ }^{3}$ Francois Roubille, ${ }^{1}$ Gilles Rioufol, ${ }^{1,2}$ Céline Giraud, ${ }^{1}$ Inesse Boussaha, ${ }^{1}$ Olivier Lairez, ${ }^{4}$ Meyer Elbaz, ${ }^{4}$ Christophe Piot, ${ }^{5,6}$ Denis Angoulvant, ${ }^{7}$ Michel Ovize ${ }^{1,2}$

To cite: Pichot S, Mewton N, Bejan-Angoulvant $\mathrm{T}$, et al. Influence of cardiovascular risk factors on infarct size and interaction with mechanical ischaemic postconditioning in STelevation myocardial infarction. Open Heart 2015;2:e000175. doi:10.1136/openhrt-2014000175

- Additional material is available. To view please visit the journal (http://dx.doi.org/ 10.1136/openhrt-2014000175).

Received 23 July 2014 Revised 24 November 2014 Accepted 13 January 2015

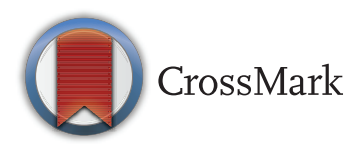

For numbered affiliations see end of article.

Correspondence to Dr Nathan Mewton; nathan.mewton@chu-lyon.fr

\section{ABSTRACT}

Objective: Previous studies have shown that mechanical postconditioning (PostC) significantly reduces infarct size (IS) in patients with acute myocardial infarction. Our objective was to assess the influence of traditional cardiovascular (CV) risk factors on IS and their interaction with ischaemic PostC in patients with acute ST-elevation myocardial infarction (STEMI).

Methods: The study population was constituted from the clinical database pooling of four previously published PostC prospective, multicentre, randomised, open-label controlled trials with identical inclusion criteria. Patients with STEMI, presenting within $12 \mathrm{~h}$ of symptoms onset referred for percutaneous coronary intervention, were included. Mechanical ischaemic PostC was performed by four repeated cycles of inflation-deflation of the angioplasty balloon within $1 \mathrm{~min}$ of reflow, while the control group underwent no intervention. IS was assessed by measuring total creatine kinase release over $72 \mathrm{~h}$.

Results: 173 patients, aged $58 \pm 12$ years, $76 \%$ males, $48 \%$ anterior infarct were included (82 in the PostC group, 91 in the control group). IS was significantly reduced in the PostC compared to the control group $\left(71.7 \pm 41.6\right.$ vs $88.2 \pm 54.5 \times 10^{3}$ arbitrary units; $p=0.027$ ). After adjustment for abnormally contracting segments, older patients had smaller IS and smokers had larger IS. Gender, diabetes, hypertension, dyslipidemia and obesity did not have any significant effect on IS. Multivariate regression analysis showed that none of the traditional risk factors had a significant impact on the cardioprotective effect of mechanical ischaemic PostC.

Conclusions: The present analysis suggests that the cardioprotective effect of mechanical PostC is not influenced by traditional CV risk factors that are prevalent in patients with STEMI.

\section{INTRODUCTION}

According to recent guidelines, coronary reperfusion should be performed as early as

\section{KEY MESSAGES}

What is already known about this subject?

- Small-size randomised trials have shown that mechanical postconditioning (PostC) significantly reduces infarct size in patients with acute myocardial infarct. However, several experimental studies suggested that comorbidities such as traditional cardiovascular risk factors had a significant impact on infarct size and interfered with PostC. These experimental results are controversial and the interaction of traditional risk factors on the cardioprotective effect of PostC in humans is scarce.

What does this study add?

- The present analysis suggests that the cardioprotective effect of mechanical PostC is not influenced by traditional cardiovascular risk factors that are prevalent in patients with ST-segment elevation myocardial infarction (STEMI).

How might this impact on clinical practice?

- Ischaemic PostC in patients with STEMI represents a new therapeutic strategy to decrease infarct size and potential clinical outcome improvement in patients with STEMI. This strategy is applicable to all patients, regardless of their comorbidities.

possible in patients presenting with ST-segment elevation myocardial infarction (STEMI) within $12 \mathrm{~h}$ of symptom onset. ${ }^{1}$ Reperfusion therapy of a jeopardised myocardium reduces the infarct size (IS) and improves the left ventricular (LV) function and clinical outcomes of patients with STEMI.

However, myocardial recovery can be suboptimal despite complete restoration of coronary blood flow, partly explained by myocardial reperfusion injury that occurs at 
the time of reperfusion. Several reports have shown the significant negative impact of reperfusion injury on $\mathrm{LV}$ remodelling, ${ }^{2}$ regional and global left ventricle recovery ${ }^{3}$ and clinical outcome. ${ }^{4}$

Developing protective strategies to reduce and prevent lethal reperfusion injury has emerged as a new therapeutic target for management of patients with STEMI. Mechanical postconditioning (PostC) consisting of brief episodes of ischaemia-reperfusion performed just after the culprit coronary artery reopening is one of these strategies. In humans, several small-sized randomised trials have shown that mechanical PostC significantly reduced IS in patients with acute myocardial infarction (AMI)..$^{5}$

The role of confounding factors on the beneficial effect of PostC remains controversial. Several experimental studies suggest that clinical characteristics such as age, ${ }^{7}$ gender $^{8}$ and traditional cardiovascular (CV) risk factors interfere with PostC. ${ }^{9}{ }^{10}$ These results are controversial $^{11} 12$ and clinical data are still lacking in humans.

Our main objective was to assess the individual influence of traditional CV risk factors on IS and their interaction with ischaemic PostC in patients with acute STEMI referred for percutaneous coronary intervention (PCI).

\section{METHODS}

\section{Study population}

The study population was constituted from the combined data set of four previously published ischaemic PostC clinical trials performed at our institution between July 2004 and October 2010. All were prospective, multicentre, randomised, open-label controlled trials. ${ }^{561314}$ These studies were pooled according to the same inclusion and exclusion criteria, and patients were submitted to the same ischaemic mechanical PostC. In the trial published by Piot et al, PostC was performed with Cyclosporine A; therefore, patients in the treatment group were not included in our pooled data set and we only included patients from the control group from this trial.

\section{Inclusion and exclusion criteria}

Briefly, patients $\geq 18$ years were included in these protocols if they presented within $12 \mathrm{~h}$ of chest pain onset with ST elevation $>0.1 \mathrm{mV}$ in two contiguous ECG leads and the clinical decision was made to treat with PCI. The culprit coronary artery had to be occluded on first coronary angiography (thrombolysis in myocardial infarction coronary flow $\leq 1$ ).

Patients with cardiac arrest, cardiogenic shock, history of AMI, angina within $48 \mathrm{~h}$ before infarction or evidence of coronary collaterals (Rentrop grade $\geq 1$ ) to the myocardial area at risk (AAR) were excluded.

All studies were performed according to the Declaration of Helsinki (revised version of Somerset West, Republic of South Africa, 1996) and according to the European Guidelines of Good Clinical Practice
(V.11, July 1990) and French laws. All patients gave written informed consent before inclusion.

\section{Area at risk}

The size of the AAR was assessed in all patients using the circumferential extent of abnormally contracting segments (ACS), according to the method of Feild et al as reported previously. ${ }^{15} 16$

\section{Experimental protocol}

The experimental protocol and primary end point assessment were the same in all four studies except the Piot et al study. Coronary angiography and PCI were performed in all patients and have been described previously. $^{5} 6$ The standard treatment after primary PCI complied with the updated European Society of Cardiology guidelines for management of STEMI patients at the time of the each trial's completion.

\section{End points}

The primary end point was IS as assessed by the area under the curve (AUC) in arbitrary units (AU) obtained by serial measurements of creatine kinase (CK) over a $72 \mathrm{~h}$ period.

30-day and 1 year rates of major adverse cardiac events were collected and assessed as a combined end point, defined as death, myocardial infarction or hospitalisation for heart failure, or any unplanned coronary revascularisation.

\section{Statistical analysis}

Data from the respective control and PostC groups from each trial were pooled in order to form one single control group and PostC group. Normality and homoscedasticity between the different studies were assessed with the Shapiro-Wilk test and Bartlett test on the first and secondary outcomes of the study. Homogeneity between the control group and the treatment groups at baseline were assessed with Student t tests for continuous variables and with $\chi^{2}$ tests for categorical variables.

The correlation between IS and the AAR was assessed in the overall population of patients and within each study group with the Spearman rank correlation analysis.

To assess the effect of specified variables on IS and their interaction with ischaemic PostC, we performed two separate multivariate robust regression models and the following variables were tested separately: age, gender, diabetes, dyslipidemia, obesity (body mass index $\geq 30 \mathrm{~kg} / \mathrm{m}^{2}$ ), hypertension and active smoking status. We used the robust multivariate linear regression model to reduce outliers' effects: the first regression assessed the individual effect of explicative variables on IS adjusting for study number, AAR and PostC effect; the second regression analysis assessed the interaction with ischaemic PostC, including the interaction between PostC effect and the explicative variable of interest. 
Clinical outcomes were compared by logistic regression analysis at 1 month and 1 year of follow-up between the control group and the PostC group.

A $p$ value $<0.05$ was considered statistically significant. All analyses were performed using R 3.0.2 software.

\section{RESULTS}

\section{Study population}

From July 2004 to October 2010, 173 patients were enrolled in the four different studies and included in the final analysis. The average age of patients was 58 \pm 12 years, $76.3 \%$ were males, $48 \%$ had an anterior infarct. Eighty-two patients were included in the ischaemic PostC group and 91 in the control group. There were no significant baseline demographic differences between groups and the baseline clinical and interventional characteristics are reported in table 1. Medications received before and during hospitalisation and at discharge are detailed in online supplementary files.

\section{Infarct size}

The AUC of serum CK release during the first $72 \mathrm{~h}$ of reperfusion was significantly reduced in the PostC group compared with the control group $\left(88.2 \times 10^{3} \pm 54.5 \times 10^{3}\right.$ $\mathrm{AU}$ vs $71.7 \times 10^{3} \pm 41.6 \times 10^{3}$ AU respectively; $\mathrm{p}=0.027$; figure 1).

AAR assessed by the ACS averaged $34.8 \pm 12.5 \%$ in the control group versus $40.3 \pm 12.3 \%$ in the PostC group $(\mathrm{p}=0.007)$. There was a significant correlation between serum CK release and AAR (Spearman's rank correlation 0.31 ; $p$ value $9.2 \times 10^{-5}$ ). For any given size of AAR, there was a significantly smaller IS in the PostC group compared with controls (figure 2).

\section{Effect of traditional CV risk factors on IS}

After adjustment on ACS, there was a significant negative relationship between age and IS ( $\beta$ coefficient -0.8 $(-1.4 ;-0.2), \mathrm{p}=0.005)$, showing a significant decrease in IS for each year gained. There was also a significant effect in smokers who had a larger IS compared to nonsmokers $(\beta$ coefficient $17.2 \quad(3.3 ; 31.2), \quad \mathrm{p}=0.02)$. Individually, gender, diabetes, hypertension, dyslipidemia and obesity did not have any significant effect on IS (table 2).

\section{Interaction of traditional CV risk factors and the effect of PostC}

The robust multivariate regression analyses showed that none of the traditional risk factors had a significant interaction with the cardioprotective effect of mechanic ischaemic PostC (table 3).

\section{CV events and mortality at $\mathbf{3 0}$ days and $\mathbf{1}$ year}

At 30 days, $14(9.2 \%)$ patients had a composite outcome (10 in the control group and 4 in the PostC group; $\mathrm{p}=0.18$; table 4 ). At 1 year, 27 patients had a composite outcome (16 in the control group and 11 in the PostC group; $\mathrm{p}=0.54)$. There was no significant difference in the composite end point between the two groups.

\section{DISCUSSION}

In this study pooling data from four different prospective PostC clinical trials performed within our institution network with identical settings, we confirmed a significant IS reduction effect of mechanical PostC in patients with STEMI. We further found that ageing and active smoking had an individual significant influence on IS.

\begin{tabular}{llll} 
Table 1 Baseline clinical characteristics & & \\
\hline & Control group $(\mathbf{n}=\mathbf{9 1})$ & PostC group ( $\mathbf{n = 8 2})$ & p Value \\
\hline Age, years & $59 \pm 12$ & $57 \pm 13$ & 0.16 \\
Male sex, \% & 80.2 & 71.9 & 0.27 \\
Body mass index, $\mathrm{kg} / \mathrm{m}^{2}$ & $26.4 \pm 4.1$ & $26.6 \pm 4.5$ & 0.77 \\
Hypertension, \% & 48.3 & 39.0 & 0.28 \\
Diabetes, \% & 19.8 & 13.4 & 0.36 \\
Smoking, \% & 53.8 & 62.2 & 0.38 \\
Dyslipidemia, \% & 48.4 & 46.3 & 0.91 \\
Admission haemodynamics & & & 0.24 \\
$\quad$ Heart rate, bpm & $71 \pm 16$ & $74 \pm 18$ & 0.48 \\
Systolic blood pressure, mm Hg & $130 \pm 26$ & $133 \pm 27$ & 0.32 \\
Ischaemia time, min & $259 \pm 140$ & $283 \pm 181$ & 0.06 \\
LV and coronary angiography & $54 \pm 11$ & $50 \pm 12$ & 0.05 \\
LV ejection fraction, \% & 13.2 & 3.7 & 0.96 \\
Multivessel status, \% & 52.7 & 51.2 & $0.007^{*}$ \\
Anterior infarct, \% & $34.8 \pm 12.5$ & $40.3 \pm 12.3$ & 0.05 \\
ACS, \% & 40.7 & 25.6 & NA \\
Thromboaspiration, \% & 100 & & \\
Direct stenting, \% & & & \\
\hline Data are presented as a percentage or as the mean $\pm S D$. & & \\
*Statistically significant. & & \\
ACS, abormality coronary segments; LV, left ventricular; PostC, postconditioning. & &
\end{tabular}


Figure 1 Assessment of infarct size by biomarker measurement. Serum creatine kinase (CK) release over the first $72 \mathrm{~h}$ of reperfusion. Area under the curve (AUC; arbitrary units) of serum CK release was measured in control (black) and postconditioned (grey) patients. There was a significant $19 \%$ reduction in the AUC of $\mathrm{CK}$ release for postconditioned versus control $(p=0.027)$.

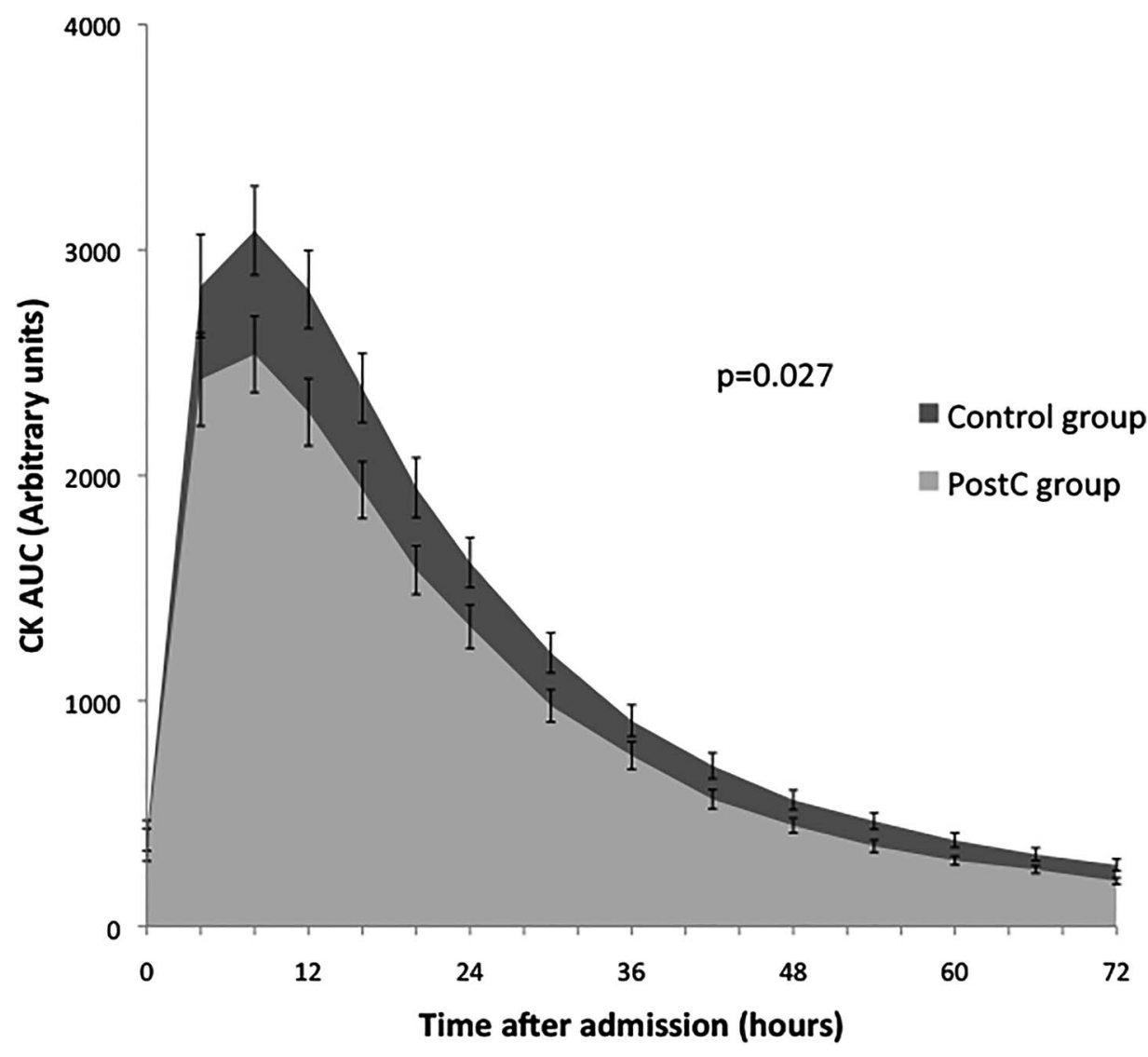

Finally, our data suggest that major CV risk factors do not significantly affect the beneficial effect of mechanical PostC on IS.

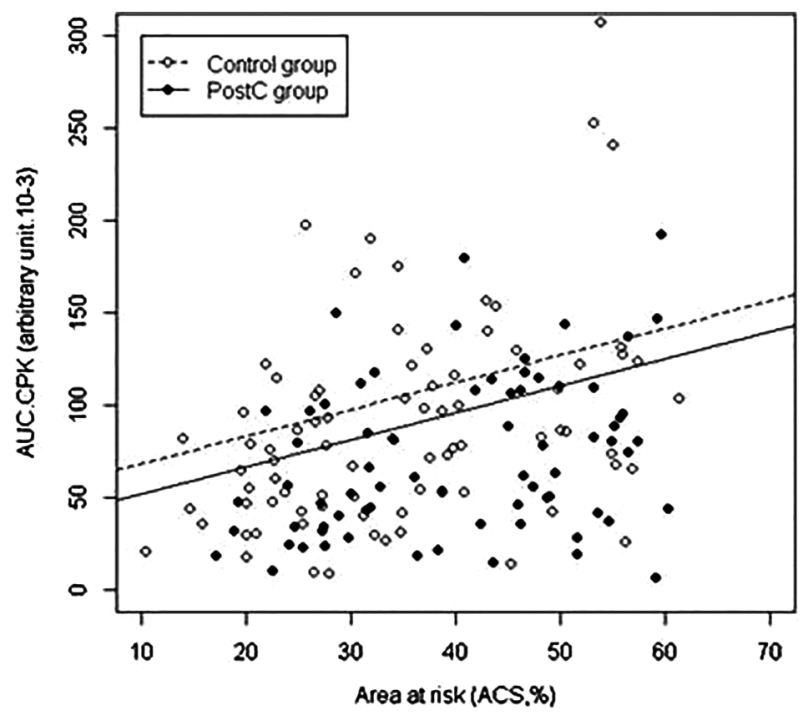

Figure 2 Reduction of cardiac enzyme release by ischaemic postconditioning (PostC), adjusted on the area at risk (AAR). Infarct size, assessed by the area under the curve of serum creatine kinase release was plotted versus abnormally contracting segments, a surrogate marker of the size of the area at risk. The line for the PostC group lies significantly below the line for the control group. In both groups, the infarct size correlates with the AAR.
Several small clinical trials have shown that applying ischaemic PostC at the time of reperfusion significantly reduced the cardiac enzyme release by up to $40 \% .^{56} 14$ IS reduction by PostC persists at 6 months. ${ }^{6}$ In a recent meta-analysis of 10 randomised trials including 560 patients, Zhou et $a l^{17}$ confirmed the significant reduction of IS by PostC. This is also concordant with a recent review by Heusch. ${ }^{18}$

Very few studies have assessed the interaction of traditional CV risk factors and demographic characteristics on PostC effect in humans. Only one recent meta-analysis of 10 randomised clinical PostC trials in patients with STEMI $^{17}$ assessed the interaction of traditional CV risk factors with ischaemic PostC. In this meta-analysis, Zhou et al showed that only gender, age and use of direct stenting had a significant interaction with PostC. The effect of PostC was more pronounced among young and male patients, and among those in whom direct stenting techniques were used. Our results on individual patient data do not confirm this significant interaction with age and gender. Furthermore, we could not assess the effect of direct stenting due to our trials protocol design where direct stenting was the only revascularisation technique applied. These differences can be explained by differences in study populations, sample size and type of analysis. Zhou et al included various study populations and different PostC protocols in their analysis. Also, their analyses were not performed on raw data from these different studies. In comparison, our 
Table 2 Effect of cardiovascular risk factors on infarct size by robust multivariate regression analysis

\begin{tabular}{lcll}
\hline & $\boldsymbol{\beta}$ Coefficient & & \\
& $\left(\times 10^{3}\right.$ AU) & 95\% Cl & p Value \\
\hline Age & -0.8 & $(-1.4$ to -0.2$)$ & $0.005^{*}$ \\
Male gender & 6.3 & $(-9.4$ to 21.9$)$ & 0.43 \\
Hypertension & -12.5 & $(-25.8$ to 0.8$)$ & 0.07 \\
Dyslipidemia & 0.05 & $(-13.1$ to 13.2$)$ & 0.99 \\
Smoking & 17.2 & $(3.3$ to to 31.2$)$ & $0.02^{*}$ \\
Diabetes & -8.2 & $(-25.8$ to -9.5$)$ & 0.37 \\
Obesity & -7.1 & $(-23.7$ to 9.6$)$ & 0.41 \\
\hline
\end{tabular}

$\beta$ Coefficient: regression coefficient estimated by robust regression adjusting for the study number, treatment group and area at risk size.

analysis presents results with a single PostC protocol as well as a complete analysis over all available raw data.

In our analysis, older age was associated with a significant reduction in IS without causing a concomitant reduction of ischaemic PostC. This might be related to a higher frequency of coronary multivessel disease and collateral blood flow in older patients. The effect of age on myocardial response to ischaemia and reperfusion is complex. Experimental studies show that older cardiomyocytes have a decreased tolerance to stress associated with decreased mitochondrial and contractile function and an increased susceptibility to apoptosis and necrosis. $^{19}$

In our study, active smokers had signficantly larger infarcts compared to non-smokers. Smoking contributes to the pathogenesis of coronary artery disease by several mechanisms including endothelial disturbance, changes in fibrin formation and turnover, altered blood rheology, changes in lipids and lipoproteins and reduced availability of antioxidants. Some ischaemia-reperfusion experimental studies have reported an increase in myocardial IS after tobacco exposure in rats. ${ }^{20}$ In humans, Robinson

Table 3 Interaction of cardiovascular risk factors with postconditioning (PostC) effect by robust multivariate linear regression

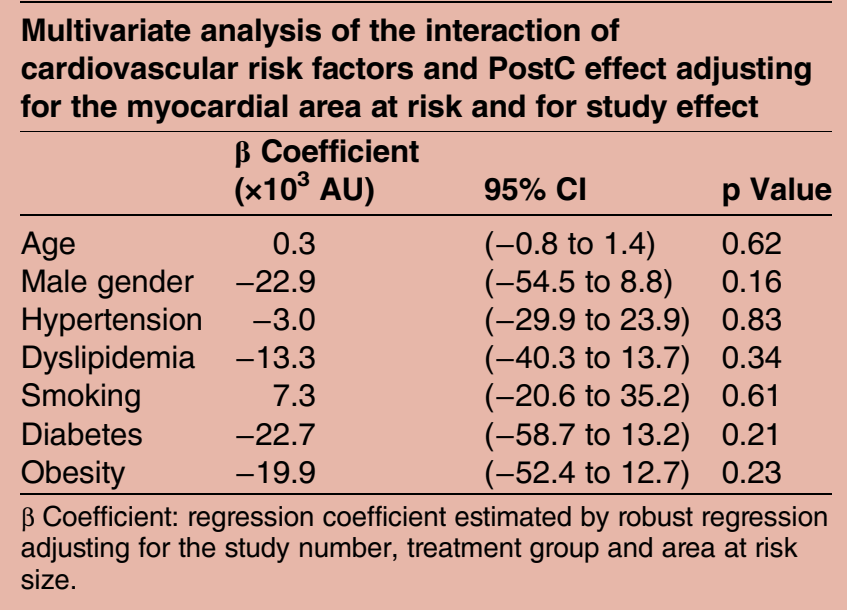

et $a l^{21}$ also showed that smokers had higher peak cardiac enzyme concentrations following an acute coronary syndrome. The effect of cigarette smoking on the cardioprotective pathways remains poorly explored. However, we did not observe any impact of smoking on the protective effect of ischaemic preconditioning (PC).

Another finding of our study is that traditional CV risk factors did not seem to have a significant influence on the protective effect of PostC. The interference of different traditional factors on PostC effect was suggested by several experimental studies. These studies had controversial findings in different ischaemia-reperfusion experimental settings. Iliodromitis $e t a l^{10}$ reported that hypercholesterolaemia significantly hampered IS reduction by ischaemic PostC, while Donato et a $t^{22}$ did not find any significant confounding effect. Similarly, ischaemic PostC did not significantly reduce IS in spontaneously hypertensive rats, ${ }^{23}$ but was protective in rats with pressure overload-induced LV hypertrophy. ${ }^{24}$ This underscores the limits of experimental models in trying to reproduce the full picture of acute ischaemic syndromes in humans. It also shows the differences that might be related to differences in species.

Diabetes is a well-known risk factor for CV morbidity and mortality after myocardial infarction in the literature. However, this higher mortality rate cannot be explained by differences in IS, determined either by biomarkers release ${ }^{25}$ or by MRI. ${ }^{26}$ The complexity of this subject has recently been emphasised with studies showing that an increase in IS is associated with hyperglycaemia on admission and its significant association with adverse outcomes. ${ }^{26}$ Experimental studies have suggested that diabetes and hyperglycaemia may have a direct detrimental effect on ischaemic myocardium and alter endothelial microvascular function. High glucose levels and diabetes are associated with increases in reactive oxygen species generation and induce impaired myocardial oxygen consumption. In experimental models, diabetes was associated with a significant loss of the ischaemic PostC cardioprotective effect. ${ }^{27}$ However, there are very little clinical data on this specific subject ${ }^{17}$ and the interaction remains to be further explored in human patients. Our study did not show any significant interaction between diabetes and ischaemic PostC.

Ischaemic PostC in patients with STEMI represents a new therapeutic strategy to decrease IS and improve clinical outcome in patients with STEMI. However, the level of evidence and the technical requirements of mechanical PostC limit its applicability to all patients with STEMI. A significant number of patients cannot benefit from this intervention which relies on a primary PCI strategy. Mechanical PostC is also difficult to combine with recent technical modifications such as thrombus aspiration. The clinical benefits of PostC techniques have yet to be demonstrated in large clinical trials. Trials with clinical end points are underway (eg, Cyclosporine and Prognosis in Acute Myocardial Infarction Patients (CIRCUS), ClinicalTrials.gov 
Table 4 Cardiovascular events and mortality at 1 year of follow-up

\begin{tabular}{|c|c|c|c|c|}
\hline $\begin{array}{l}\text { Composite end point } \\
30 \text { days }\end{array}$ & $\begin{array}{l}\text { Total cohort } \\
14(9.2 \%)\end{array}$ & $\begin{array}{l}\text { Control group } \\
10(12.5 \%)\end{array}$ & $\begin{array}{l}\text { PostC group } \\
4(5.5 \%)\end{array}$ & $\begin{array}{l}p \text { Value } \\
0.18\end{array}$ \\
\hline 1 year & $27(19.3 \%)$ & $16(21.6 \%)$ & $11(16.7 \%)$ & 0.54 \\
\hline All-cause mortality & 2 & 0 & 2 & \\
\hline Hospitalisation for heart failure & 10 & 7 & 3 & \\
\hline Unstable angina & 2 & 1 & 1 & \\
\hline Myocardial infarction & 3 & 3 & 0 & \\
\hline Unplanned revascularisation & 13 & 7 & 5 & \\
\hline
\end{tabular}

Identifier: NCT01502774A) and will bring important findings on the place of PostC strategies in patients with STEMI. Of note, comorbidities and traditional CV risk factors might not necessarily have a similar influence on pharmacological than on ischaemic PostC.

\section{Study limitations}

First, the sample size was small and was constituted by pooling populations of four different clinical trials realised within our multicentre network at different time points. The limited size of our study sample, as well as a posteriori statistical analysis, limits our power to discriminate any effect that could exist from one of the CV risk factors studied.

We had full access to all clinical databases from these trials and all these clinical trials shared the same PostC protocol and same exclusion and inclusion criteria. We tested the heterogeneity between the different studies by comparing population baseline characteristics between each study and we did not find any significant difference. During the different inclusion periods from July 2004 to October 2010, significant changes in international guidelines and therapeutic management of patients with STEMI occurred, and could have induced a potential bias of confounding factors.

Finally, we found a significant difference for the AAR measure between the PostC and control groups in the pooled population. This difference, however, is in favour of the control group, the ACS being significantly greater in the PC group. In all previous trials, there was a consistent trend of the ACS being larger in the PC group, although not reaching significance. The pooling of the individual data from each one of these trials and the addition of the control group from the Piot et al trial made this difference significant. However, we do not think this affects the results and conclusions presented in our manuscript.

\section{CONCLUSION}

In this study, we confirmed the significant IS reduction effect by mechanical PostC in patients with STEMI. The cardioprotective effect of mechanical PostC is not influenced by traditional CV risk factors that are prevalent in patients with STEMI. These trials will also provide further data increasing the statistical power to assess the interaction between postconditioning interventions and patients baseline characteristics such as diabetes, age and all the potential effect modifiers that have been described in the experimental literature. They will also provide further data on the relationship between patient's characteristics and PostC techniques efficiency.

\section{Author affiliations}

${ }^{1}$ Cardiology Division, Hôpital Cardiologique Louis Pradel, Centre

d'Investigation Clinique, Hospices Civils de Lyon, Lyon, France

${ }^{2}$ Inserm UMR-1060, CarMeN Unit, Université Claude Bernard Lyon1, Lyon, France

${ }^{3}$ CHRU Tours, Service de Pharmacologie, Hôpital Bretonneau; CNRS UMR 7292, Université François Rabelais, GICC, Tours, France

${ }^{4}$ Hôpital Rangueuil, Université Paul Sabatier, Toulouse, France

${ }^{5}$ Hopital Arnaud de Villeneuve, Université de Montpellier I and II, Montpellier, France

${ }^{6}$ Inserm U661, Montpellier, France

${ }^{7} \mathrm{CHRU}$ Tours, Hôpital Trousseau, Université François Rabelais EA 4245, Tours, France

Contributors All the authors contributed to refinement of the study protocol and approved the final manuscript and patients recruitment. CG, TB-A and SP were responsible for design, analysis and interpretation of data. SP, NM and MO was involved in proof writing and MO is the guarantor.

Funding This project was supported by a research grant from the Hospices Civils de Lyon and from the ministry of health.

Competing interests None declared.

Patient consent Obtained.

Provenance and peer review Not commissioned; externally peer reviewed.

Open Access This is an Open Access article distributed in accordance with the Creative Commons Attribution Non Commercial (CC BY-NC 4.0) license, which permits others to distribute, remix, adapt, build upon this work noncommercially, and license their derivative works on different terms, provided the original work is properly cited and the use is non-commercial. See: http:// creativecommons.org/licenses/by-nc/4.0/

\section{REFERENCES}

1. Boersma E, Maas AC, Deckers JW, et al. Early thrombolytic treatment in acute myocardial infarction: reappraisal of the golden hour. Lancet 1996;348:771-5.

2. Ganame J, Messalli G, Dymarkowski S, et al. Impact of myocardial haemorrhage on left ventricular function and remodelling in patients with reperfused acute myocardial infarction. Eur Heart $J$ 2009;30:1440-9.

3. Nijveldt R, Beek AM, Hirsch A, et al. Functional recovery after acute myocardial infarction: comparison between angiography, electrocardiography, and cardiovascular magnetic resonance measures of microvascular injury. J Am Coll Cardiol 2008;52:181-9.

4. Bolognese L, Carrabba N, Parodi G, et al. Impact of microvascular dysfunction on left ventricular remodeling and long-term clinical 
outcome after primary coronary angioplasty for acute myocardial infarction. Circulation 2004;109:1121-6.

5. Staat P, Rioufol G, Piot C, et al. Postconditioning the human heart. Circulation 2005;112:2143-8.

6. Thibault $\mathrm{H}$, Piot $\mathrm{C}$, Staat $\mathrm{P}$, et al. Long-term benefit of postconditioning. Circulation 2008;117:1037-44.

7. Przyklenk K, Maynard M, Darling CE, et al. Aging mouse hearts are refractory to infarct size reduction with post-conditioning. J Am Coll Cardiol 2008;51:1393-8.

8. Penna C, Tullio F, Merlino A, et al. Postconditioning cardioprotection against infarct size and post-ischemic systolic dysfunction is influenced by gender. Basic Res Cardiol 2009;104:390-402.

9. Bouhidel O, Pons S, Souktani R, et al. Myocardial ischemic postconditioning against ischemia-reperfusion is impaired in ob/ob mice. Am J Physiol Heart Circ Physiol 2008;295:H1580-6.

10. Iliodromitis EK, Zoga A, Vrettou A, et al. The effectiveness of postconditioning and preconditioning on infarct size in hypercholesterolemic and normal anesthetized rabbits. Atherosclerosis 2006;188:356-62.

11. Yin $\mathrm{Z}$, Gao H, Wang $\mathrm{H}$, et al. Ischaemic post-conditioning protects both adult and aged Sprague-Dawley rat heart from ischaemia-reperfusion injury through the phosphatidylinositol 3-kinase-AKT and glycogen synthase kinase-3beta pathways. Clin Exp Pharmacol Physiol 2009;36:756-63.

12. Przyklenk K, Ovize M, Bauer B, et al. Gender does not influence acute myocardial infarction in adult dogs. Am Heart $J$ 1995;129:1108-13.

13. Piot $\mathrm{C}$, Croisille $\mathrm{P}$, Staat $\mathrm{P}$, et al. Effect of cyclosporine on reperfusion injury in acute myocardial infarction. $N$ Engl $J$ Med 2008;359:473-81.

14. Thuny $\mathrm{F}$, Lairez $\mathrm{O}$, Roubille $\mathrm{F}$, et al. Post-conditioning reduces infarct size and edema in patients with ST-segment elevation myocardial infarction. J Am Coll Cardiol 2012;59:2175-81.

15. Alderman EL. The angiographic definitions of the Bypass Angioplasty Revascularization Investigation. Coron Artery Dis 1992;12:1189-208.

16. Feild BJ, Russell ROJ, Dowling JT, et al. Regional left ventricular performance in the year following myocardial infarction. Circulation 1972;46:679-89.
17. Zhou C, Yao Y, Zheng Z, et al. Stenting technique, gender, and age are associated with cardioprotection by ischaemic postconditioning in primary coronary intervention: a systematic review of 10 randomized trials. Eur Heart $J$ 2012;33:3070-7.

18. Heusch G. Cardioprotection: chances and challenges of its translation to the clinic. Lancet 2013;381:166-75.

19. Bernhard D, Laufer $G$. The aging cardiomyocyte: a mini-review. Gerontology 2008;54:24-31.

20. Zhu BQ, Sun YP, Sievers RE, et al. Exposure to environmental tobacco smoke increases myocardial infarct size in rats. Circulation 1994;89:1282-90

21. Robinson K, Conroy RM, Mulcahy R. Smoking and acute coronary heart disease: a comparative study. $\mathrm{Br}$ Heart $\mathrm{J}$ 1988;60:465-9.

22. Donato M, D'Annunzio V, Berg G, et al. Ischemic postconditioning reduces infarct size by activation of $A 1$ receptors and $\mathrm{K}+(\mathrm{ATP})$ channels in both normal and hypercholesterolemic rabbits. $J$ Cardiovasc Pharmacol 2007;49:287-92.

23. Penna C, Tullio F, Moro F, et al. Effects of a protocol of ischemic postconditioning and/or captopril in hearts of normotensive and hypertensive rats. Basic Res Cardiol 2010;105:181-92.

24. Zhu M, Feng J, Lucchinetti E, et al. Ischemic postconditioning protects remodeled myocardium via the PI3K-PKB/Akt reperfusion injury salvage kinase pathway. Cardiovasc Res 2006;72:152-62.

25. Hasdai D, Granger CB, Srivatsa SS, et al. Diabetes mellitus and outcome after primary coronary angioplasty for acute myocardial infarction: lessons from the GUSTO-Ilb Angioplasty Substudy. Global use of strategies to open occluded arteries in acute coronary syndromes. J Am Coll Cardiol 2000;35:1502-12.

26. Eitel I, Hintze S, de Waha S, et al. Prognostic impact of hyperglycemia in nondiabetic and diabetic patients with ST-elevation myocardial infarction: insights from contrast-enhanced magnetic resonance imaging. Circ Cardiovasc Imaging 2012;5:708-18.

27. Zhu SG, Xi L, Kukreja RC. Type 2 diabetic obese $\mathrm{db} / \mathrm{db}$ mice are refractory to myocardial ischaemic post-conditioning in vivo: potential role for Hsp20, F1-ATPase delta and Echs1. J Cell Mol Med 2012;16:950-8. 